\title{
Curativo gel de clorexidina no transplante de células-tronco hematopoéticas
}

Chlorhexidine gel dressing in hematopoietic stem cell transplantation

Vendaje gel de clorhexidina en el trasplante de células madre hematopoyéticas

Lais Esparrachiari Carvalho Castanho ${ }^{1}$ ib https://orcid.org/0000-0001-9126-5589

Bruna Nogueira dos Santos ${ }^{1}$ id https://orcid.org/0000-0002-1939-6194

Amanda Salles Margatho ${ }^{1}$ io https://orcid.org/0000-0002-1006-9357

Fernanda Titareli Merizio Martins Braga ${ }^{1}$ id https:///rerid.org/0000-0001-8089-788X

Paula Elaine Diniz dos Reis ${ }^{2}$ ib https://orcid.org/0000-0002-9782-3366

Maria Carolina de Oliveira ${ }^{3}$ id https://orcid.org/0000-0003-0691-2222

Renata Cristina de Campos Pereira Silveira ${ }^{1}$ id https://orcid.org/0000-0002-2883-3640

Como citar:

Castanho LE, Santos BN, Margatho AS, Braga FT, Reis PE, Oliveira MC, et al. Curativo gel de clorexidina no transplante de célulastronco hematopoéticas. Acta Paul Enferm. 2020;33:eAPE20180307.

DOI

http://dx.doi.org/10.37689/actaape/2020A00307

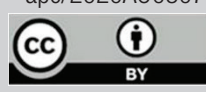

Descritores

Tansplante de células-tronco hematopoéticas; Infecções relacionadas a cateter; Cateteres venosos centrais; Clorexidina; Curativos

hidrocoloides

Keywords

Hematopoietic stem cell transplantation; Catheterrelated infections; Central venous catheters; Chlorhexidine; Bandages, hydrocolloid

Descriptores

Trasplante de células madre hematopoyéticas; Infecciones relacionadas con catéteres; Catéteres venosos centrales; Clorhexidina; Vendas

hidrocoloidales

Submetido 2 de Janeiro de 2019

Aceito

16 de Outubro de 2019

Autor correspondente

Bruna Nogueira dos Santos

E-mail: brunandsantos@hotmail.com

\section{Resumo}

Objetivo: Acompanhar o uso do curativo gel de clorexidina em cateter venoso central de crianças e adultos submetidos ao transplante de células-tronco hematopoéticas para verificar a ocorrência de infecção da corrente sanguínea relacionada ao cateter e irritação cutânea na vigência deste curativo.

Métodos: Estudo observacional e prospectivo, com 25 pacientes com cateter venoso central inserido para realização do transplante de células-tronco hematopoéticas. Diariamente avaliou-se as características do curativo, da pele, clínicas do paciente e relacionadas a infecção. Os pacientes foram acompanhados a partir do primeiro dia da inserção do CVC até a sua remoção, até o limite de 45 dias a partir da data de inserção do CVC.

Resultados: A infecção da corrente sanguínea relacionada ao cateter ocorreu em 28\% dos pacientes, houve associação entre neutropenia febril e infecção $(p<0,01)$. A irritação cutânea foi identificada em $24 \%$ dos pacientes. Não foi identificada associação entre infecção da corrente sanguínea relacionada ao cateter e a irritação cutânea $(\mathrm{p}=0,51)$. Houve associação significativa entre irritação cutânea e suspensão do uso do curativo $(p=0,03)$. As trocas não programadas ocorreram em $50 \%$ das trocas identificadas e 0 principal motivo foi a presença de sangue no curativo $(57,8 \%)$.

Conclusão: 0 uso do curativo gel de clorexidina em pacientes submetidos ao TCTH demonstrou ser uma medida eficaz na reduçãa da ocorrência de infecções relacionadas ao cateter, quando comparado aos dados da literatura. Os casos de ICSRC encontrados foram associados, principalmente, à condição de neutropenia, muito comum nesta população. Os casos de irritação cutânea relacionados ao curativo identificados e a presença de sangue como principal motivo de troca não programada salientam a importância do conhecimento relacionado ao uso do curativo por parte dos enfermeiros assistenciais, a fim de criar protocolos que indiquem o uso e manejo do curativo com segurança.

\section{Abstract}

Objective: Monitor the use of chlorhexidine gel dressing in the central venous catheter of children and adults submitted to hematopoietic stem cell transplantation (HSCT) to check for catheter-related bloodstream infection and skin irritation while using this dressing.

Methods: This is a prospective observational study with 25 patients with central venous catheters (CVC) inserted for hematopoietic stem cell transplantation. Dressing characteristics, skin characteristics, clinical conditions of patients and infection-related characteristics were evaluated daily. Patients were monitored from the first day of CVC insertion until removal, up to 45 days from the CVC insertion date.

Results: Catheter-related bloodstream infection (CRBSI) occurred in $28 \%$ of all patients, with an association between febrile neutropenia and infection $(p<0.01)$. Skin irritation was identified in $24 \%$ of patients. No 
association was found between catheter-related bloodstream infection and skin irritation $(p=0.51)$. A significant association was observed between skin irritation and dressing removal $(p=0.03)$. Unscheduled dressing changes corresponded to $50 \%$ of all dressing changes identified during the study, and the main reason was presence of blood in the dressing (57.8\%).

Conclusion: Using chlorhexidine gel dressing in patients submitted to HSCT proved to be an effective measure to reduce the occurrence of catheter-related infections, when compared to literature data. The cases of CRBSI found in this study were mainly associated with the condition of neutropenia, which is very common in this population. The cases of dressing-related skin irritation and presence of blood as the main reason for unscheduled change highlight the importance of nurses having proper knowledge about how to use this dressing in order to create protocols for safe dressing use and handling.

\section{Resumen}

Objetivo: Hacer un seguimiento del uso del vendaje gel de clorhexidina en catéter venoso central (CVC) en niños y adultos sometidos al trasplante de células madre hematopoyéticas (TCMH) para verificar los casos de infección del flujo sanguíneo relacionados con el catéter (IFSRC) y la irritación cutánea durante la validez de este vendaje.

Métodos: Estudio observacional y prospectivo, con 25 pacientes con catéter venoso central insertado para realizar el trasplante de células madre hematopoyéticas. Diariamente se evaluaron las características del vendaje y de la piel, las características clínicas del paciente y las relacionadas con la infección. Los pacientes recibieron un seguimiento a partir del primer día de la inserción del CVC hasta su retirada, con un límite de 45 días a partir de la fecha de inserción del CVC.

Resultados: La infección del flujo sanguíneo relacionada con el catéter se presentó en el 28\% de los pacientes, hubo relación entre neutropenia febril e infección $(p<0,01)$. Se identificó irritación cutánea en el $24 \%$ de los pacientes. No se identificó relación entre la infección del flujo sanguíneo relacionada con el catéter y la irritación cutánea $(p=0,51)$. Hubo relación significativa entre la irritación cutánea y la suspensión del uso del vendaje ( $p=0,03)$. Los cambios no programados sucedieron en el $50 \%$ de los cambios registrados, y el motivo principal fue la presencia de sangre en el vendaje $(57,8 \%)$.

Conclusión: El uso del vendaje gel de clorhexidina en pacientes sometidos al TCMH demostró ser una medida eficaz para la reducción de casos de infecciones relacionadas con el catéter, en comparación con los datos de la literatura. Los casos de IFSRC encontrados se relacionaron principalmente con la condición de neutropenia, muy común en esta población. Los casos identificados de irritación cutánea relacionados con el vendaje y la presencia de sangre como principal motivo de cambio no programado resaltan la importancia del conocimiento del uso del vendaje por parte de los enfermeros asistenciales, a fin de crear protocolos que indiquen el uso y manejo seguro del vendaje.

\section{Introdução}

O Transplante de Células-Tronco Hematopoéticas (TCTH) consiste na infusão endovenosa de célulastronco hematopoéticas com finalidade de restabelecer a função da medula óssea, sendo indicado no tratamento de doenças malignas e não malignas. ${ }^{(1)}$ Para facilitar o manejo dos pacientes e assegurar a infusão de medicamentos com segurança, os pacientes elegíveis ao TCTH necessitam de um Cateter Venoso Central (CVC) instalado antes do início do regime de condicionamento com quimioterápicos, que precede o TCTH. ${ }^{(2)}$

Durante o TCTH, utilizam-se, geralmente, CVC permanentes ou temporários. O CVC permanente é tunelizado, como o cateter do tipo Hickman, implantado de forma cirúrgica por um trajeto subcutâneo, denominado túnel, e um cuff na porção distal impregnado por antimicrobiano. ${ }^{(2,3)} \mathrm{O}$ CVC temporário, não tunelizado, é inserido percutaneamente em veia central, através de procedimento realizado no leito do paciente, tal como o cateter duplo lúmen. ${ }^{(3,4)}$

Embora seja um dispositivo essencial, o CVC não é isento de complicaçôes, dentre as quais se destaca a Infecção da Corrente Sanguínea Relacionada ao Cateter (ICSRC), que consiste em fator preditivo para a remoção do cateter entre pacientes submetidos ao TCTH, ${ }^{(3,5,6)}$ além de aumentar taxas de morbimortalidade, tempo de internação e custos hospitalares. ${ }^{(3,7)}$

Os pacientes submetidos ao TCTH são potencialmente mais susceptíveis às ICSRCs devido à fragilidade imunológica induzida pelo tratamento e pela própria doença de base. ${ }^{(8)}$ Além disso, a imunossupressão intensa, tempo de neutropenia prolongado, quebra da integridade cutânea para inserçáo do cateter e cicatrização retardada devido aos efeitos dos quimioterápicos contribuem para a ocorrência de complicaçóes infecciosas. ${ }^{(8-10)}$

A incidência das infecçóes relacionadas ao cateter intravascular pode ser reduzida com adoção de medidas preventivas, tais como higienizar as mãos antes de manipular o cateter e suas conexóes, usar barreira de proteção máxima durante a inserção do cateter, antissepsia da pele com clorexidina, evitar acesso em veia femoral, remover os cateteres quando esses não forem mais necessários, realizar as trocas de curativos conforme recomendação, e avaliar diariamente o sítio de saída dos cateteres, curativos e conexôes. ${ }^{(4,11)}$

Uma estratégia para redução da infecção relacionada ao cateter intravascular é a utilização de curativo com gel ou disco impregnado com clorexidina. ${ }^{(7,12)}$ Este curativo mostra-se efetivo na redução da colonização cutânea por microrganismos causadores de conta- 
minação extra luminal do cateter. ${ }^{(9,13)}$ Por outro lado, é fundamental haver protocolos bem estabelecidos para trocas de curativo, uma vez que a adesividade inerente a qualquer tipo de curativo utilizado como cobertura do CVC pode ocasionar lesóes cutâneas decorrentes da remoção do estrato córneo da pele. ${ }^{(10,14)}$

Duas revisóes sistemáticas da literatura indicaram efeito benéfico dos curativos impregnados com clorexidina, em gel ou disco, para prevenir colonização e reduzir a incidência das infecçôes relacionadas ao cateter, ${ }^{(7,12)}$ porém poucos estudos descrevem os aspectos relacionados à aplicação e à manutenção diária do curativo, ${ }^{(15,16)}$ principalmente no contexto de pacientes onco-hematológicos submetidos a altas doses de quimioterapia seguidas ou não por TCTH. ${ }^{(9,17,18)} \mathrm{O}$ curativo gel de clorexidina (CGCHX) foi identificado como superior em relação à capacidade de supressão de crescimento bacteriano. ${ }^{(19,20)}$ Como vantagens, destaca-se que o CGCHX é aplicado em uma única etapa, permite o contato direto da clorexidina a $2 \%$ com a pele e o fato do gel ser translúcido permite a visualização do sítio de saída. ${ }^{(15)}$

Este estudo teve por objetivo acompanhar o uso do CGCHX em CVC de crianças e adultos submetidos ao TCTH para verificar a ocorrência de ICSRC e irritação cutânea na vigência deste curativo.

\section{Métodos}

Trata-se de estudo observacional e prospectivo realizado nas unidades de internaçáo de Transplante de Medula Óssea, Hematologia e Terapia Imunológica e na unidade ambulatorial do Transplante de Medula Óssea do Hospital de Clínicas da Faculdade de Medicina de Ribeirão Preto da Universidade de São Paulo.

A amostra foi constituída por 25 pacientes. Foram incluídos crianças e adultos, com CVCs inseridos para realização do TCTH para tratamento de doenças autoimunes, hematológicas benignas ou onco-hematológicas. A inclusão de crianças no estudo se deu pelo fato de que o protocolo assistencial da instituição prevê utilização de curativo transparente para todos os pacientes submetidos ao TCTH.

Os critérios de exclusão foram histórico de alergia à clorexidina e ter recebido a inserção do CVC antes da admissão hospitalar. Os dados foram co- letados no período de 23 de setembro de 2013 a 23 de fevereiro de 2014. Ao atender os critérios de seleção, o paciente ou familiar responsável (quando o paciente era criança) recebeu explicaçóes sobre o estudo e foi convidado pela pesquisadora para participar da pesquisa. Todos os pacientes elegíveis atenderam aos critérios de seleção e aceitaram convite para serem incluídos no estudo. O protocolo da pesquisa está demonstrado na figura 1 .

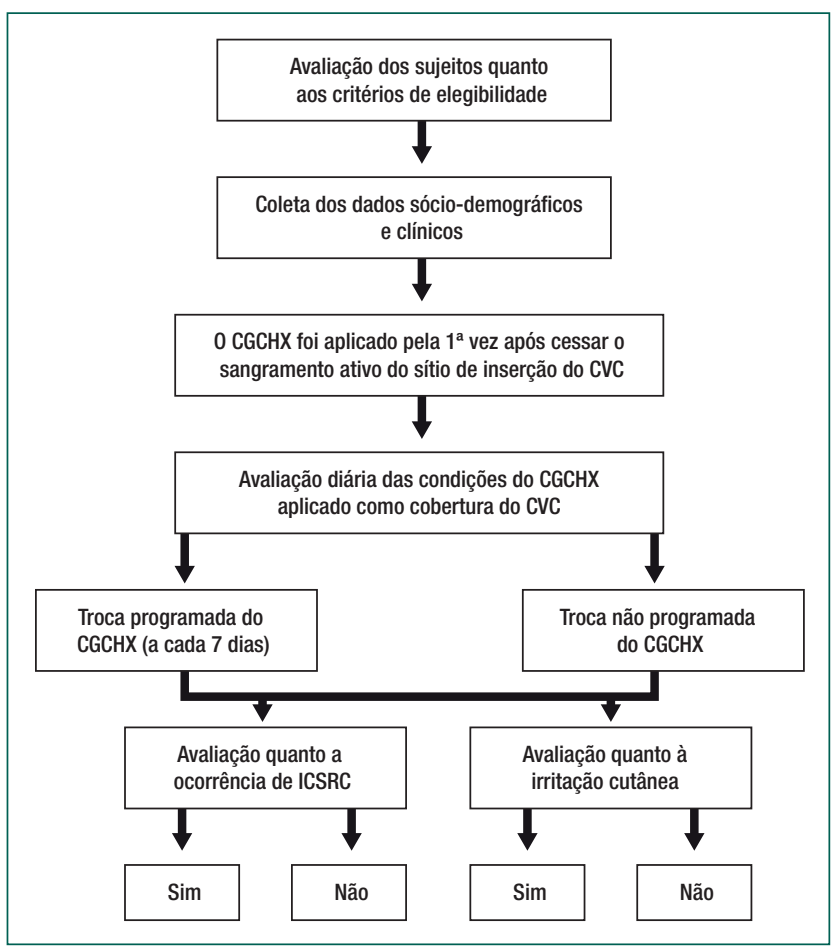

Figura 1. Fluxograma das etapas do protocolo da pesquisa

De acordo com o protocolo institucional das unidades de internação, o CGCHX (Tegaderm CHG, $3 \mathrm{M}$, Brasil) era colocado assim que cessasse o sangramento ativo no sítio de saída do CVC pós inserção, geralmente até 48 horas depois da inserção do cateter.

As trocas dos curativos dos CVC eram realizadas pelos 21 enfermeiros das unidades de internação. Antes do início da coleta de dados, o pesquisador realizou um treinamento com os 21 enfermeiros das unidades de internação a fim de padronizar o procedimento de troca do curativo. $\mathrm{O}$ treinamento consistiu numa aula expositiva específica sobre o CGCHX e a técnica de troca do mesmo, precedida por um pré-teste e sucedida por um pós-teste com 20 questóes assertivas que tiveram o objetivo de identificar o conhecimento prévio dos profissionais e o quanto foi acrescentado a este conhecimento. 
A coleta dos dados foi realizada por uma equipe de pesquisadores, composta pela pesquisadora principal e dois auxiliares de pesquisa que receberam o mesmo treinamento dos enfermeiros, acrescido de orientaçóes sobre o preenchimento do instrumento de coleta de dados utilizado no estudo.

O tempo de seguimento de 45 dias foi definido de acordo com resultados de um estudo prévio realizado em uma das unidades de internação, que identificou o tempo médio de permanência dos cateteres de 45 dias. ${ }^{(5)}$

Para a coleta de dados utilizou-se um instrumento validado quanto ao conteúdo e a aparência por cinco juízes. As análises foram feitas por meio do software SAS 9.2 e pelo software Statistical Package for the Social Science (SPSS,) versáo 25.0. Adotou-se nível de significância de 5\%. Utilizaram-se frequências absolutas e percentuais, média, desvio-padrão, valores de mediana (mínimo e máximo) e o modelo de regressão log-binomial simples e múltiplo para estimar o Risco Relativo (RR) da ocorrência de ICSRC e de irritação cutânea. $\mathrm{O}$ teste de Mann-Whitney foi empregado para comparar a ICSRC com as variáveis quantitativas de interesse. As medidas de associação foram feitas por teste exato de Fisher.

O estudo foi aprovado pelo Comitê de Ética em Pesquisa da Escola de Enfermagem de Ribeirão Preto da Universidade de São Paulo cadastrado sob o número CAAE: 12055813.7.0000.539. Os pacientes e os enfermeiros assinaram o Termo de Consentimento Livre e Esclarecido. Em relação às crianças, o termo de assentimento foi aplicado para aquelas que eram alfabetizadas e seus pais assinaram também o Termo de Consentimento Livre e Esclarecido.

O curativo avaliado já era utilizado nos locais de estudo, conforme protocolo institucional, porém estas unidades não dispunham de curativos em quantidade suficiente para uso em todos os pacientes durante todo o tratamento do TCTH. Diante disso, uma quantidade de curativos foi obtida pelas pesquisadoras por meio de compra efetuada com Recurso da Coordenação de Aperfeiçoamento de Pessoal de Nível Superior (CAPES) para garantir o andamento do estudo. Não houve conflito de interesses de qualquer pesquisador/autor do estudo.
Os desfechos primários deste estudo foram a ocorrência de ICSRC (sim ou não) e a presença de irritação cutânea relacionada ao CGCHX (sim ou não).

A ICSRC foi definida como bacteremia ou fungemia durante o uso do CVC com uma ou mais hemoculturas positivas de sangue periférico, manifestaçôes clínicas de infecção (febre, tremores e/ou hipotensão) na ausência de outra fonte de infeção identificada, com exceção do cateter, além do crescimento de microrganismo em cultura de sangue obtido do hub do cateter, detectado duas horas antes da positivação de hemocultura periférica. ${ }^{(21)}$ Esta é a definição adotada pela Comissão de Controle de Infecção Hospitalar (CCIH) do hospital que a utiliza como critério para o diagnóstico microbiológico da infecção, assim foi mantido no desenvolvimento do estudo, uma vez que os dados relacionados à ICRSRC foram obtidos junto a esta Comissão.

Para identificar irritação cutânea no local de adesão do curativo, a pele em contato com o CGCHX foi avaliada a cada troca do curativo, em busca dos sinais estabelecidos pelo Grupo de Pesquisa Internacional de Dermatite de Contato: pele normal; eritema leve; eritema e pele ligeiramente espessa ou eritema intenso com edema e vesículas. ${ }^{(22)}$ As trocas programadas ocorreram no sétimo dia de uso do curativo ${ }^{(4)} \mathrm{e}$ as não programadas antes desse período. ${ }^{(7,14,16)}$ Os pacientes que apresentaram irritação cutânea tiveram o uso do CGCHX suspenso, e foram acompanhados até o último dia de uso do curativo. Em contrapartida, aqueles que tiveram a suspensão temporária do uso do curativo devido a sangramento ativo no sítio de saída do CVC foram acompanhados até a remoção do cateter, pois assim que o sangramento cessou, o uso do CGCHX foi reiniciado. Havendo suspensão do curativo, era iniciado curativo com gaze estéril e fita adesiva. Com o intuito de evitar confusão na avaliação da irritação cutânea relacionada à adesividade do curativo, os pesquisadores inspecionaram diariamente quanto aos sinais e sintomas de Doença do Enxerto Contra o Hospedeiro (DECH) aguda da pele, segundo os seguintes estágios de severidade: grau 0 ausência de rash cutâneo; grau 1 presença de rash cutâneo acometendo área de superfície corporal $<25 \%$; grau 2 presença de rash acometendo área de superfície 
corporal entre 25 a 50\%; grau 3 presença de rash cutâneo acometendo área de superfície corporal $>50 \%$; e grau 4 eritrodermia generalizada acometendo área de superfície corporal $>50 \%$ associada à formação de bolhas e descamação da área de superfície corporal $<5 \%$. ${ }^{(23)}$

Os desfechos secundários relacionados aos CVC e a troca dos curativos foram: tipo de cateter; tempo de permanência do CVC durante o período de coleta de dados (0-45 dias); localização; motivo de retirada; tipo de microrganismo relacionado à ocorrência de ICRSC; tempo decorrido entre a inserção do CVC e o início do uso do curativo; número de trocas do curativo por paciente; quantidade de trocas programadas e não programadas; motivos de trocas dos curativos; suspensão do curativo e descolamento do curativo no sítio de saída do cateter. Outros desfechos secundários foram dados sociodemográficos e clínicos dos pacientes (idade, sexo, dias de internação, doença de base, tipo de TCTH, tipo de regime de condicionamento quimioterápico, dias para início da quimioterapia após inserção do CVC, dias para enxertia da medula óssea, neutropenia na admissão hospitalar e neutropenia febril).

$\mathrm{O}$ regime de condicionamento quimioterápico foi classificado por alta, média e reduzida intensidade, segundo os seguintes critérios: alta intensidade, regime de condicionamento mieloablativo com pancitopenia irreversível; média intensidade, regime de condicionamento não mieloablativo, com mínima pancitopenia; e baixa intensidade, regime de condicionamento que não pode ser classificado como de alta ou média intensidade. ${ }^{(24)}$

Considerou-se enxertia da medula óssea quando o paciente apresentou três dias consecutivos com contagem absoluta de neutrófilos no sangue acima de $500 / \mathrm{mm}^{3}$ após o TCTH. ${ }^{(25)}$

A variável neutropenia na admissão hospitalar indica que, ao iniciar o TCTH, o paciente já se encontrava neutropênico (contagem absoluta de neutrófilos no sangue menor que $1.000 / \mathrm{mm}^{3}$ ). ${ }^{(26)}$ Neutropenia febril foi definida como a contagem absoluta de neutrófilos menor do que $1.000 / \mathrm{mm}^{3}$ e uma única medida de temperatura maior do que $38,3^{\circ} \mathrm{C}$ ou temperatura maior ou igual a $38^{\circ} \mathrm{C}$ sustentada por mais de 1 hora. ${ }^{(27)}$

\section{Resultados}

A amostra foi constituída por 25 pacientes. A tabela 1 descreve as características sociodemográficas e clínicas dos pacientes. A caracterização dos cateteres e troca de curativo também estão apresentadas na tabela 1 .

Tabela 1. Características demográficas, clínicas, relacionadas aos cateteres e às trocas dos curativos $(n=25)$

\begin{tabular}{|c|c|}
\hline Características & $n(\%)$ \\
\hline $\begin{array}{l}\text { Idade em anos } \\
\text { Mediana (min;max) }\end{array}$ & $32(4 ; 66)$ \\
\hline \multicolumn{2}{|l|}{ Sexo } \\
\hline Feminino & $13(52)$ \\
\hline Masculino & $12(48)$ \\
\hline Dias de internação, média (DP) & $34(15,1)$ \\
\hline Mediana (min; max) & $34(12 ; 81)$ \\
\hline \multicolumn{2}{|l|}{ Doença de base } \\
\hline Leucemia & $7(28)$ \\
\hline Mieloma múltiplo & $5(20)$ \\
\hline Linfoma & $4(16)$ \\
\hline Outras $^{*}$ & $9(36)$ \\
\hline Dias para enxertia, média (DP) & $16(5,3)$ \\
\hline Mediana (min; max) & $14(11 ; 30)$ \\
\hline \multicolumn{2}{|l|}{ Tipo d } \\
\hline Autólogo & $16(64)$ \\
\hline Alogênico & $9(36)$ \\
\hline \multicolumn{2}{|l|}{ Intensidade do regime de condicionamento } \\
\hline Alta intensidade & $12(48)$ \\
\hline Média intensidade & $5(20)$ \\
\hline Intensidade reduzida & 8(32) \\
\hline Início do regime de condicionamento, média (DP) & $2(2,56)$ \\
\hline Mediana (min; max) & $1(0 ; 10)$ \\
\hline \multicolumn{2}{|l|}{ Neutropenia na admissão hospitalar } \\
\hline Sim & $7(28)$ \\
\hline Não & $18(72)$ \\
\hline \multicolumn{2}{|l|}{ Neutropeni febril } \\
\hline Sim & $13(52)$ \\
\hline Não & $12(48)$ \\
\hline \multicolumn{2}{|l|}{ Tipo de CVC } \\
\hline Duplo lúmen & $14(56)$ \\
\hline Hickman & $11(44)$ \\
\hline Tempo de permanência do CVC, média (DP) & $26(14,3)$ \\
\hline Mediana (min; max) & $19(10 ; 45)$ \\
\hline \multicolumn{2}{|l|}{ Local de acesso } \\
\hline Veia jugular interna & $20(80)$ \\
\hline Veia subclávia & $5(20)$ \\
\hline \multicolumn{2}{|l|}{ Motivo retirada CVC } \\
\hline Fim tratamento & $9(36)$ \\
\hline Suspeita de infecção relacionada ao cateter & $8(32)$ \\
\hline Cateter não integro & $2(8)$ \\
\hline Remoção involuntária do cateter & $1(4)$ \\
\hline Cateter não retirado (duração de mais de 45 dias) & $5(20)$ \\
\hline Trocas do curativo por paciente, média (DP) & $3(1,9)$ \\
\hline Mediana (min; max) & $3(1 ; 7)$ \\
\hline
\end{tabular}

DP - desvio padrão; min - valor mínimo; max - valor máximo; *0utras - lúpus eritematoso sistêmico ( $n=1)$, esclerose sistêmica $(n=2)$, síndrome de Poems $(n=1)$, anemia aplásica/falciforme $(n=3)$, síndrome mielo displásica $(n=1)$, mielofibrose $(n=1)$ 
Em 32\% ( $n=8)$ dos pacientes, os regimes de condicionamento foram iniciados um dia após a inserção do CVC e, em quatro (16\%), sete ou mais dias após a colocação do CVC.

A ocorrência de ICSRC foi identificada em $28 \%(n=7)$ dos pacientes, sendo que em dois casos esta complicação foi tratada e não implicou na remoção do cateter. A análise microbiológica das amostras identificou cinco bactérias gram negativas (Chryseobacterium indologenes, Acinetobacter baumannii, Escherichia coli, Klebsiella pneumoniae e Comamonas testosteroni) e duas gram positivas (Staphylococcus epidermidis e Corynebacterium jk).

$\mathrm{O}$ intervalo de tempo médio entre a inserção do cateter e o início do uso do CGCHX foi de dois dias $(60 \%, n=15)$, variando entre um e sete dias. Os CGCHX aplicados quatro dias após a inserção do cateter completaram, em maior frequência, sete dias de permanência, ou seja, foram trocados no tempo programado.

Foram realizadas 90 trocas de curativos, sendo $50 \%(n=45)$ programadas e $50 \%$ não programadas. $\mathrm{O}$ principal motivo de troca não programada foi a presença de sujidade no curativo $(57,8 \%, \mathrm{n}=26)$, seguido de curativo descolado $(31,1 \%, \mathrm{n}=14)$, curativo não integro $(6,7 \%, n=3)$ e irritação cutânea $(4,4 \%, n=2)$.

Do total da amostra, apenas um paciente (4\%) desenvolveu DECH de pele aguda grau 1 durante o período de coleta de dados, cuja complicação iniciou 30 dias após o transplante, no $43^{\circ}$ dia após a inserção do CVC. O paciente apresentou prurido e rash cutâneo em tórax, que regrediram em poucos dias após início do tratamento para $\mathrm{DECH}$ e não implicou na suspensão do uso do CIGCHX.

Dos pacientes em estudo, 24\% ( $n=6)$ apresentaram irritação cutânea na pele em contato com o CGCHX. Destes, três tiveram seus curativos suspensos permanentemente. Outros dois pacientes tiveram suspensão do uso do curativo por sangramento ativo no sítio de saída do cateter, retomando o uso posteriormente. Na avaliação cutânea durante os procedimentos de troca dos curativos observouse a presença de eritema leve em $88 \%(n=22)$ dos pacientes em alguma das trocas.

Nas tabelas 2 e 3 é apresentado respectivamente o risco relativo da ocorrência de ICSRC e de irritação cutânea de acordo com as variáveis elencadas.

Tabela 2. Risco Relativo (RR) de infecção de corrente sanguínea relacionada ao cateter

\begin{tabular}{|c|c|c|c|c|c|}
\hline \multirow[b]{2}{*}{ Variável } & \multicolumn{2}{|c|}{ ICSRC } & \multirow[b]{2}{*}{$\begin{array}{l}\text { Total } \\
\mathrm{n}(\%)\end{array}$} & \multirow{2}{*}{$\begin{array}{l}\text { Risco Relativo } \\
\text { (IC 95\%) }\end{array}$} & \multirow[b]{2}{*}{$p$-value } \\
\hline & $\begin{array}{l}\text { Sim } \\
\mathrm{n}(\%)\end{array}$ & $\begin{array}{l}\text { Não } \\
\mathrm{n}(\%)\end{array}$ & & & \\
\hline \multicolumn{6}{|l|}{ Neutropenia febril } \\
\hline Sim & $7(54)$ & $6(46)$ & $13(100)$ & - & $<0,01^{*}$ \\
\hline Não & 0 & $12(100)$ & $12(100)$ & & \\
\hline \multicolumn{6}{|l|}{ Neutropenia na admissão, n (\%) } \\
\hline Sim & 2(29) & $5(71)$ & $7(100)$ & $1,03(0,26-4,12)$ & $0,97^{\star *}$ \\
\hline Não & $5(28)$ & $13(72)$ & 18(100) & 1 & \\
\hline \multicolumn{6}{|l|}{ Tipo de CVC } \\
\hline Hickman & $3(27)$ & $8(73)$ & $11(100)$ & $0,95(0,27-3,40)$ & $0,94^{\star \star}$ \\
\hline Duplo lúmen & $4(29)$ & $10(71)$ & 14(100) & 1 & \\
\hline \multicolumn{6}{|c|}{ Regime de condicionamento quimioterápico } \\
\hline alta intensidade & $4(33)$ & $8(67)$ & $12(100)$ & $2,67(0,36-19,71)$ & $0,34^{\star *}$ \\
\hline intensidade média & $2(40)$ & $3(60)$ & $5(100)$ & $3,20(0,38-26,78)$ & $0,28^{\star \star}$ \\
\hline intensidade reduzida & $1(13)$ & $7(87)$ & $8(100)$ & 1 & \\
\hline \multicolumn{6}{|l|}{ Irritação cutânea } \\
\hline Sim & $1(17)$ & $5(83)$ & $6(100)$ & $0,53(0,08-3,56)$ & $0,51^{\star \star}$ \\
\hline Não & $6(32)$ & $13(68)$ & 19(100) & 1 & \\
\hline \multicolumn{6}{|l|}{ Tipo de TCTH } \\
\hline Alogênico & 2(22) & $7(78)$ & $9(100)$ & 1 & \\
\hline Autólogo & $5(31)$ & $11(69)$ & $16(100)$ & $1,41(0,34-5,83)$ & $0,64^{\star *}$ \\
\hline Dias para a Enxertia, média (DP) & $18,6(5,8)$ & $15,6(4,9)$ & $25(100)$ & $1,06(0,98-1,15)$ & $0,17^{\star \star \star}$ \\
\hline $\begin{array}{l}\text { Trocas Não Programadas } \\
\text { média (DP) }\end{array}$ & $1,3(1,2)$ & $2,0(1,2)$ & $25(100)$ & $0,68(0,39-1,19)$ & $0,18^{\star \star \star}$ \\
\hline
\end{tabular}

ICSRC - infecção de corrente sanguínea relacionada ao cateter; DP - desvio-padrão; *Não foi possível calcular a estimativa do risco relativo. 0 valor-p calculado se refere ao teste exato de Fisher; *^0 p-value calculado se refere ao teste exato de Fisher; ${ }^{\star \star \star} 0$ valor-p calculado se refere ao teste de Mann-Whitney 
Tabela 3. Risco Relativo (RR) de irritação cutânea

\begin{tabular}{|c|c|c|c|c|c|}
\hline \multirow[b]{2}{*}{ Variável } & \multicolumn{2}{|c|}{ Presença de irritação cutânea } & \multirow[b]{2}{*}{$\begin{array}{l}\text { Total } \\
\text { n(\%) }\end{array}$} & \multirow[b]{2}{*}{$\begin{array}{l}\text { Risco Relativo } \\
\text { (IC 95\%) }\end{array}$} & \multirow[b]{2}{*}{$p$-value } \\
\hline & $\underset{\mathrm{n}(\%)}{\mathrm{Sim}}$ & $\begin{array}{l}\text { Não } \\
\text { n(\%) }\end{array}$ & & & \\
\hline \multicolumn{6}{|l|}{ Neutropenia febril } \\
\hline Sim & $3(23)$ & $10(77)$ & $13(100)$ & $0,92(0,23-3,72)$ & 0,91 \\
\hline Não & $3(25)$ & $9(75)$ & $12(100)$ & 1 & \\
\hline \multicolumn{6}{|l|}{ Neutropenia na admissão } \\
\hline Sim & $3(43)$ & $4(57)$ & $7(100)$ & $2,57(0,67-9,83)$ & 0,17 \\
\hline Não & $3(17)$ & $15(83)$ & $18(100)$ & 1 & \\
\hline \multicolumn{6}{|l|}{ Tipo de CVC } \\
\hline Hickman & $3(27)$ & $8(73)$ & $11(100)$ & $1,27(0,32-5,12)$ & 0,73 \\
\hline Duplo lúmen & $3(21)$ & $11(79)$ & $14(100)$ & 1 & \\
\hline \multicolumn{6}{|c|}{ Regime de condicionamento quimioterápico } \\
\hline alta intensidade & $3(25)$ & $9(75)$ & $12(100)$ & $1,00(0,21-4,71)$ & 0,99 \\
\hline intensidade média & $1(20)$ & $4(80)$ & $5(100)$ & $0,80(0,10-6,7)$ & 0,84 \\
\hline intensidade reduzida & $2(25)$ & $6(75)$ & $8(100)$ & 1 & \\
\hline \multicolumn{6}{|l|}{ Suspensão do CGCHX } \\
\hline Sim & $3(60)$ & $2(40)$ & $5(100)$ & $4,00(1,13-14,17)$ & 0,03 \\
\hline Não & $3(15)$ & $17(85)$ & $20(100)$ & 1 & \\
\hline \multicolumn{6}{|l|}{ Tipo de TCTH } \\
\hline Alogênico & $3(33)$ & $6(67)$ & $9(100)$ & 1 & \\
\hline Autólogo & $3(19)$ & $13(81)$ & $16(100)$ & $0,56(0,14-2,23)$ & 0,41 \\
\hline
\end{tabular}

${ }^{*} p$-value calculado se refere ao teste exato de Fisher

\section{Discussão}

A inserção do CVC é considerada essencial para realização do TCTH, facilitando o procedimento e aumentando sua segurança. Entretanto, sua instalação rompe a integridade cutânea, abrindo uma porta de entrada aos microrganismos patogênicos ${ }^{(28)}$ e representa a principal fonte de infecção em pacientes submetidos ao TCTH. ${ }^{(29)}$ No presente estudo, foram utilizados dois tipos de cateteres: duplo lúmen (56\%, n=14) e Hickman (44\%, n=11). Na literatura, o CVC tunelizado é considerado uma estratégia que inibe a migração de microrganismos para o trajeto do cateter, reduzindo as taxas de ICSRC. (4) No presente estudo, no entanto, não foi encontrada associação entre o tipo de CVC utilizado9 e a ocorrência de ICSRC (RR=0,95; IC=0,27-3,40; $\mathrm{p}=0,94)$.

Em 28\% ( $n=7)$ dos pacientes, identificaram-se ICSRCs com maior crescimento de bactérias gramnegativas. As infecçóes causadas por bactérias gramnegativas associam-se a maior gravidade e mortalidade, ${ }^{(30,31)}$ porém tais resultados contrastam com os achados da literatura internacional que evidenciam maiores crescimentos de bactérias gram- positivas. $(9,12,17,32)$ Estudos epidemiológicos ${ }^{(30,31)}$ demonstram que a presença de neutropenia e imunossupressão são fatores associados para a ocorrência de ICSRC causadas por bactérias gram-negativas.

Fatores como o tipo de TCTH, neutropenia na admissão hospitalar, tipo de condicionamento quimioterápico e tempo para enxertia da medula estão associadas ao desenvolvimento de infecçóes, ${ }^{(8)}$ porém, neste estudo, não foram encontradas associaçôes destes fatores com o risco de desenvolvimento de ICSRC. No transplante alogênico, o paciente possui maior risco de complicaçôes infecciosas, enquanto nos pacientes submetidos ao transplante autólogo, o risco é menor. ${ }^{(8)} \mathrm{A}$ imunossupressão induzida após o transplante alogênico para evitar a rejeição do enxerto é um desses fatores. A intensidade do regime de condicionamento que difere entre os tipos de transplante influencia no tempo da enxertia. Quanto maior o tempo para enxertia da medula, maior o tempo de neutropenia e, consequentemente, maior o risco de desenvolver infecçóes. ${ }^{(8)}$

A neutropenia identificada na admissão hospitalar pode estar associada a fatores pré-transplante, como terapias imunossupressoras prévias e a própria doença. ${ }^{(8)}$ Neste estudo, a neutropenia febril apresentou associação estatisticamente significante à ocorrência de ICSRC $(\mathrm{p}<0,01)$. A neutropenia pode ser uma manifestação clínica da doença de base, devido à substituição das células normais da medula óssea por 
células neoplásicas. ${ }^{(28)}$ A neutropenia é o principal fator de risco para o desenvolvimento de infecçóes relacionadas ao CVC e frequente causa de morte nesta população. ${ }^{(8)}$ Além da adoção de medidas preventivas, a incidência das ICSRC pode ser reduzida por meio da escolha adequada do curativo para cobertura do sítio de saída do cateter. O CGCHX tem sido recomendado para pacientes com alta suscetibilidade a infecçóes, incluindo os neutropênicos. ${ }^{(16)}$

Embora haja evidências sobre a efetividade do curativo de clorexidina na redução da incidência de ICSRC em pacientes onco-hematológicos ${ }^{(9,17)}$ ou naqueles sob cuidados intensivos, ${ }^{(7,12)}$ é preciso avaliar sua indicação e permanência quando há irritação cutânea em seu local de aplicação, por se tratar de um curativo adesivo. Dentre os pacientes avaliados neste estudo, 24\% (n=6) apresentaram irritação cutânea quando em uso do CGCHX, e o curativo foi suspenso em $12 \%(n=3)$ dos pacientes em decorrência da irritação cutânea. Não foi encontrada associação significativa $(\mathrm{p}=0,51)$ entre a ocorrência de irritação cutânea e o desenvolvimento de ICSRC nos pacientes em estudo.

A presença de sangue (curativo sujo) foi o principal motivo da remoção não programada do curativo $(57,8 \%)$, fato este que evidencia a importância do conhecimento das características peculiares da clientela, como o tempo de exsudação sanguinolenta do sítio de saída do cateter e a interferência do tempo entre a inserção do cateter e o início do condicionamento quimioterápico. ${ }^{(10,33)}$ A literatura aponta que deve ser respeitado o período de sete dias após procedimentos invasivos, tal como a inserção de CVC, para início de ciclos quimioterápicos, o que pode amenizar a influência dos agentes quimioterápicos sobre o processo de cicatrização e sangramento do local de inserção do cateter. ${ }^{(33)}$

Os CGCHX foram aplicados em média dois dias após a inserção do cateter, assim que evidenciado ausência de sangramento ativo no sítio de saída do cateter como recomendado pela literatura. ${ }^{(4)}$ No entanto, em alguns casos, o sítio de saída apresentou sangramento posterior, implicando na remoção do curativo, inclusive com necessidade de suspensão temporária do seu uso. Este fato pode estar relacionado à influência do início precoce do ciclo quimioterápico após a inserção do cateter e à plaquetopenia. Condutas podem ser tomadas para minimizar esse problema, tais como respeitar o período de sete dias após o implante do cateter para o início do ciclo quimioterápico, transfusóes sanguíneas ou até mesmo o uso de cola cirúrgica no sítio de saída do cateter para reduzir sangramento. ${ }^{(16,34)}$

$\mathrm{O}$ segundo motivo de troca mais frequente foi o descolamento do curativo $(31,1 \%)$. Tal resultado pode estar relacionado a condiçóes frequentemente observadas nesta população, tais como neutropenia febril, que pode levar à sudorese excessiva durante os picos febris, fazendo com que os curativos descolem com maior facilidade. ${ }^{(14,16)} \mathrm{O}$ descolamento do curativo é um fator de risco para as infecçóes relacionadas ao cateter. ${ }^{(14)}$

Como limitaçóes deste estudo descreve-se o tamanho amostral pequeno e, devido ao delineamento proposto neste estudo, a impossibilidade de estabelecer relação causa e efeito entre o curativo gel de clorexidina e ocorrência de infecção relacionada ao cateter intravascular. Além disso, os resultados são aplicáveis a uma população muito específica, ou seja, aos pacientes submetidos ao TCTH.

E dentre as contribuiçóes deste estudo para a prática clínica envolvem o conhecimento do uso de uma nova tecnologia de cobertura do sítio de saída do cateter venoso central e o manejo da sua aplicação diária. Desta forma, descreve os cuidados de enfermagem imprescindíveis para manutenção segura deste curativo, a fim de evitar a ocorrência de eventos adversos como as infecçóes relacionadas ao cateter e a irritação cutânea causada pela adesividade do curativo em uma população específica e frágil como o paciente submetido ao TCTH. Para futuras pesquisas sugerimos a avaliação da neutropenia não apenas na admissão hospitalar, mas em outros momentos pontuais do período de internação do paciente, visto que este é o principal fator de risco para sangramento na inserção do cateter venoso em pacientes oncológicos.

\section{Conclusão}

Este estudo corrobora com os achados da literatura que recomendam o uso do curativo gel de clorexidina para pacientes neutropênicos, ou seja, mais susceptíveis a complicaçóes infecciosas, tendo em vista que 
esta é uma medida eficaz para reduzir a ocorrência de infecçóes relacionadas ao cateter. Consideramos necessário que centros de transplantes desenvolvam protocolos de condutas para indicação, manutenção e troca deste curativo, com intuito de otimizar o seu uso, tendo em vista que esta é uma medida eficaz para reduzir a ocorrência de ICSRC. Dessa forma, a adoção de práticas que garantam a permanência do CGCHX e minimizem o risco de irritação cutânea é fundamental para a assegurar a segurança do cuidado ao paciente bem como contribuir para a adequada qualidade de vida durante o tratamento. Neste estudo, verificou-se que não houve associação entre presença de irritação cutânea e a ocorrência de ICSRC.

\section{Agradecimentos}

À Coordenação de Aperfeiçoamento de Pessoal de Nível Superior (CAPES; bolsa de mestrado para Laís Esparrachiari Carvalho Castanho - PROEX 0552/2018 - Processo 23038.000945/2018-37); ao Programa de Pós-Graduação Enfermagem Fundamental da Escola de Enfermagem de Ribeirão Preto, Universidade de São Paulo pelo financiamento parcial do estudo; aos profissionais das unidades de Transplante de Medula Óssea, Hematologia e Terapia Imunológica do Hospital das Clínicas da Faculdade de Medicina de Ribeirão Preto que contribuíram para a execução deste trabalho.

\section{Colaborações}

Castanho LEC, Santos BN, Margatho AS, Braga FTMM, Reis PED, Oliveira MC e Silveira RCCP declaram que contribuíram com a concepção do projeto, análise e interpretação dos dados, revisão crítica relevante do conteúdo intelectual e aprovação da versão final a ser publicada.

\section{Referências}

1. Li HW, Sykes M. Emerging concepts in haematopoietic cell transplantation. Nat Rev Immunol. 2012;12(6):403-16.
2. Rodrigues HF, Garbin LM, Castranho LC, Simoes BP, Curcioli AC, Silveira $\mathrm{RC}$. [Hickman catheters in hematopoietic stem cell transplantation: surgical implantation, removal and nursing care]. Rev Enferm UERJ. 2015;23(3):304-9. Portuguese.

3. Centers for Diseases Control and Prevention (CDC). Bloodstream infection event (central line-associated bloodstream infection and non-central line associated bloodstream infection). CDC; 2018. Available from: https:// www.cdc.gov/nhsn/pdfs/pscmanual/4psc_clabscurrent.pdf

4. O'Grady NP, Alexander M, Burns LA, Dellinger EP, Garland J, Heard SO, et al. Guidelines for the prevention of intravascular catheter-related infections. Am J Infect Control. 2011; 39(4 Suppl 1):1-34.

5. Castanho LC, Silveira RC, Braga FT, Canini SR, Reis PE, Voltarelli JC. [Rationale for Hickman catheter removal in patients undergoing hematopoietic stem cell transplantation] Acta Paul Enferm. 2011;24:244-8. Portuguese.

6. Yilmaz MC, Aksoylar S, Erdogan D, Demirag B. Complications of central venous catheters in children undergoing hematopoietic stem cell transplantation in Turkey. J Pediatr Oncol Nurs. 2012;29(4):199-205.

7. Ullman AJ, Cooke ML, Mitchell M, Lin F, New K, Long DA, et al. Dressings and securement devices for central venous catheters (CVC). Cochrane Database Syst Rev. 2015; (9):CD010367.

8. Tomblyn M, Chiller T, Einsele H, Gress R, Sepkowitz K, Storek J, et al. Guidelines for preventing infectious complications among hematopoietic cell transplant recipients: a global perspective. Biol Blood Marrow Transplant. 2009;44(8):453-5.

9. Biehl LM, Huth A, Panse J, Krämer C, Hentrich M, Engelhardt M, et al. A Randomized trial on chlorhexidine dressings for the prevention of catheter-related bloodstream infections in neutropenic patients. Ann Oncol. 2016;27(10):1916-22.

10. Benhamou E, Fessard E, Com-Nougue C, Beaussier PS, Nitenberg G, Tancrede $\mathrm{C}$, et al. Less frequent catheter dressing changes decrease local cutaneous toxicity of high-dose chemotherapy in children, without increasing the rate of catheter-related infections: results of a randomised trial. Bone Marrow Transplant. 2002;29(8):653-8.

11. Marschall J, Mermel LA, Fakih M, Hadaway L, Kallen A, O'Grady NP, et al. Strategies to prevent central line-associated bloodstream infections in acute care hospitals: 2014 update. Infect Control Hosp Epidemiol. 2014;35(7):753-71.

12. Safdar N, O'Horo JC, Ghufran A, Bearden A, Didier ME, Chateau $D$, et al. Chlorhexidine-impregnated dressing for prevention of catheter-related bloodstream infection: a meta-analysis*. Crit Care Med. 2014;42(7):1703-13.

13. Karpanen TJ, Casey AL, Whitehouse T, Nightingale P, Das I, Elliott TSJ. Clinical evaluation of a chlorhexidine intravascular catheter gel dressing on short-term central venous catheters. Am J Infect Control. 2016;44(1):54-60.

14. Timsit JF, Bouadma L, Ruckly S, Schwebel C, Garrouste-Orgeas $\mathrm{M}$, Bronchard $\mathrm{R}$, et al. Dressing disruption is a major risk factor for catheter-related infections. Crit Care Med. 2012;40(6):1707-14.

15. Pfaff $B$, Heithaus T, Emanuelsen M. Use of a 1-peace chlorhexidine gluconate transparent dressing on critically ill patients. Crit Care Nurse. 2012;32(4):35-40.

16. Jeanes A, Bitmead J. Reducing bloodstream infection with a chlorhexidine gel IV dressing. Br J Nurs. 2015;24(19):S14-9.

17. Chambers ST, Sanders J, Patton WN, Ganly P, Birch M, Crump JA, et al. Reduction of exit-site infections of tunnelled intravascular catheters among neutropenic patients by sustained-release chlorhexidine dressings: results from a prospective randomized controlled trial. J Hosp Infect. 2005;61(1):53-61. 
18. Santos BN, Oliveira MC, Braga FT, Margatho AS, Esparrachiari LC, Silveira RC. Local cutaneous effects associated with chlorhexidineimpregnated gel dressing in hematopoietic stem cell transplantation patients. Open J Nurs. 2018;8(2):115-29.

19. Bashir MH, Olson LK, Walters SA. Suppression of regrowth of normal skin flora under chlorhexidine gluconate dressings applied to chlorhexidine gluconate-prepped skin. Am J Infect Control. 2012; 40(4):344-8.

20. Kawamura H, Takahashi N, Takahashi M, Taketomi A. Differences in microorganism growth on various dressings used to cover injection sites: inspection of the risk of catheter-related bloodstream infections caused by Gram-negative bacilli. Surg Today. 2014;44(12):2339-44.

21. Mermel LA, Allon M, Bouza E, Craven DE, Flynn P, O'Grady NP, et al. Clinical practice guidelines for the diagnosis and management of intravascular catheter-related infection: 2009 Update by the Infectious Diseases Society of America. Clin Infect Dis. 2009;49(1):1-45.

22. Wilkinson DS, Fregert S, Magnusson B, Bandmann HJ, Calnan CD, Cronin E, et al. Terminology of contact dermatitis. Acta Derm Venereol. 1970;50(4):287-92.

23. Schoemans HM, Lee SJ, Ferrara JL, Wolff D, Levine JE, Schultz KR, et al. EBMT-NIH-CIBMTR Task Force position statement on standardized terminology \& guidance for graft-versus-host disease assessment. Bone Marrow Transplant. 2018;53(11):1401-15.

24. Bacigalupo A, Ballen K, Rizzo D, Giralt S, Lazarus H, Ho V, et al. Defining the intensity of conditioning regimens: working definitions. Biol Blood Marrow Transplant. 2009;15(12):1628-33.

25. Popat U, Mehta RS, Rezvani K, Fox P, Kondo K, Marin D, et al. Enforced fucosylation of cord blood hematopoietic cells accelerates neutrophil and platelet engraftment after transplantation. Blood. 2015;125(19):2885-92.

26. Centers for Disease Control and Prevention; Infectious Disease Society of America; American Society of Blood and Marrow Transplantation.
Guidelines for preventing opportunistic infections among hematopoietic stem cell transplant recipientes. MMWR Recomm Rep. 2000 0ct;49(RR-10):1-125, CE1-7. Erratum in: MMWR Recomm Rep. 2004;53(19):396.

27. U.S. Department of Health and Human Services, National Institutes of Health, National Cancer Institute. Common terminology criteria for adverse events (CTCAE) Version 5.0. 2017:3. Avaliable from: https:// ctep.cancer.gov/protocolDevelopment/electronic_applications/docs/ CTCAE_v5_Quick_Reference_8.5x11.pdf

28. Khayr W, Haddad RY, Noor SA. Infections in hematological malignancies. Dis Mon. 2012;58(4):239-49.

29. Gudiol C, Garcia-Vidal C, Arnan M, Sánchez-Ortega I, Patiño B, Duarte $\mathrm{R}$, et al. Etiology, clinical features and outcomes of pre-engraftment and post-engraftment bloodstream infection in hematopoietic SCT recipients. Bone Marrow Transplant. 2014;49(6):824-30.

30. Braun E, Hussein K, Geffen Y, Rabino G, Bar-Lavie Y, Paul M. Predominance of Gram-negative bacilli among patients with catheterrelated bloodstream infections. Clin Microbiol Infect 2014;20(10):06279.

31. Hajjej Z, Nasri M, Sellami W, Gharsallah H, Labben I, Ferjani M. Incidence, risk factors and microbiology of central vascular catheterrelated bloodstream infection in an intensive care unit. J Infect Chemother. 2014;20(3):163-8.

32. Ruschulte H, Franke M, Gastmeier P, Zenz S, Mahr KH, Buchholz S, et al. Prevention of central venous catheter related infections with chlorhexidine gluconate impregnated wound dressings: a randomized controlled trial. Ann Hematol. 2009;88(3):267-72.

33. Payne WG, Naidu DK, Wheeler CK, Barkoe D, Mentis M, Salas RE, et al. Wound healing in patients with cancer. Eplasty. 2008;8:9.

34. Pittiruti M, Scoppettuolo G, Emoli A, Musarò A, Biasucci D. Cyanoacrylate glue and central venous access device insertion [abstract]. JAVA. 2016; 21(4):249. 